\title{
Imunologia da retinocoroidite toxoplásmica
}

\author{
Immunology of the toxoplasmic retinochoroiditis
}

Cynthia Azeredo Cordeiro ${ }^{1,2}$, Paula Rocha Moreira ${ }^{3}$, Walderez Ornelas Dutra ${ }^{3}$, Lucy Young ${ }^{1}$, Wesley Ribeiro Campos $^{2}$, Fernando Oréfice², ANTÔNIO LÚCIO TEIXEIRA JÚNIOR ${ }^{4}$

\section{RESUMO}

A infecção pelo Toxoplasma gondii é uma importante causa de doença ocular, tanto em indivíduos imunocomprometidos como em imunocompetentes. A patogênese da destruição retinocoroidiana associada a essa infecção ainda não está totalmente esclarecida. Nesta revisão, discute-se o papel do sistema imune no controle da infecção pelo Toxoplasma, especialmente, no olho.

Descritores: Toxoplasmose ocular/imunologia; Coriorretinite; Uveíte; Toxoplasma/imunologia;Autoimunidade/imunologia;Linfócitos Treguladores/imunologia; Citocinas/imunologia

\section{ABSTRACT}

Toxoplasma gondii infection is an important cause of ocular disease in both immunocompromised and immunocompetent subjects. The pathogenesis of retinochoroidal lesion associated with this infection is not fully understood. In this review, the role of the imm une system in the control of Toxoplasma infection, especially in the eye, is discussed.

Keywords: Toxoplasmosis, ocular/immunology; Chorioretinitis; Uveitis; Toxoplasma/immunology; Autoimmunity/immunology; T-lymphocytes, regulatory/ immunology; Cytokines/immunology

\section{INTRODUÇÃO}

A toxoplasmose é uma zoonose causada pelo Toxoplasma gondii, um parasita intracelular obrigatório, com ampla distribuição geográfica e alta prevalência sorológica mundial(1). No Brasil, a prevalência de infecção por toxoplasmose em adultos varia de 50 a $80 \%$ dependendo da região estudada ${ }^{(1-2)}$. A infecção pode ser congênita por via transplacentária ou adquirida, ocorrendo através da ingestão ou manipulação de carne crua ou mal-passada contendo cistos teciduais, ou água ou comida contendo oocistos eliminados em fezes de gatos infectados ${ }^{(3-4)}$.

A infecção toxoplásmica em humanos é frequentemente subclínica, ou seja, apresenta-se assintomática ou com sintomas inespecíficos, como os de um resfriado comum, sem determinar qualquer comprometimento ocular. No entanto, pode tornar-se clinicamente evidente, evoluindo, por exemplo, com retinocoroidite em indivíduos imunossuprimidos ou não. A retinocoroidite toxoplásmica é reconhecidamente a causa mais comum de uveíte posterior e pode deixar sequelas graves, incluindo a perda completa da visão(2,5-7).

Os fatores que controlam a ocorrência, a gravidade e a recorrência da doença ocular não são bem compreendidos, embora uma variedade de componentes, incluindo a susceptibilidade genética do hospedeiro, o estado nutricional e do

Trabalho realizado na Universidade Federal de Minas Gerais - UFMG - Belo Horizonte (MG), Brasil.

Retina Department, Massachusetts Eye and Ear Infirmary, Harvard Medical School, Boston, MA, EUA.

Departamento de Oftalmologia, Setor de Uveítes, Hospital São Geraldo, Universidade Federal de Minas Gerais - UFMG - Belo Horizonte (MG), Brasil.

${ }^{3}$ Departamento de Morfologia, Instituto de Ciências Biológicas, Universidade Federal de Minas Gerais - UFMG - Belo Horizonte (MG), Brasil.

${ }^{4}$ Departamento de Clínica Médica, Faculdade de Medicina, Universidade Federal de Minas ${ }^{4}$ Departamento de Clínica Médica, Faculdade de
Gerais - UFMG - Belo Horizonte (MG), Brasil.

Endereço para correspondência: Cynthia A. Cordeiro. Beacon Street - 1595 - Apto. 12 Brookline, MA 02445 EUA - E-mail: cordeiro.cy@gmail.com

Recebido para publicação em 06.12.2008

Última versão recebida em 15.09.2010

Aprovação em 17.10.2010 sistema imune, a carga parasitária, o genótipo do parasita têm sido sugeridos como possivelmente envolvidos no desenvolvimento da infecção $0^{(8-9)}$.

Muitas questões sobre a doença permanecem ainda intrigando oftalmologistas e infectologistas. A resposta à antibioticoterapia combinada ou não com corticóides varia amplamente entre os pacientes. A apresentação clínica também é bastante heterogênea, sendo que alguns pacientes apresentam episódio de inflamação mínima, enquanto outros têm múltiplas recorrências de uveíte grave, levando à perda da visão(6,10). A resposta imune provavelmente apresenta um papel relevante na evolução da doença e possivelmente na resposta à terapia convencional ${ }^{(10)}$. Neste artigo, fazemos uma revisão sobre a resposta imune na toxoplasmose, com ênfase no acometimento ocular da doença.

\section{RESPOSTA IMUNE NA TOXOPLASMOSE}

O Toxoplasma gondii, parasita intracelular, induz uma resposta imune consistente e duradoura que busca o controle da proliferação dos taquizoítos. A resposta imune inata, representada por macrófagos, células "natural killer" (NK) e células polimorfonucleares atuam na primeira linha de defesa do organismo ${ }^{(11)}$.

\section{IMUNIDADE INATA}

O parasita estimula diretamente os macrófagos a produzirem citocinas como interleucina (IL)-1 $\beta, \mathrm{IL}-12$ e fator de necrose tumoral $\alpha$ (TNF- $\alpha$ ). Essas citocinas atuam em sinergia para induzirem as células NK a produzirem interferon $\Upsilon$ (IFN- $\Upsilon$ ). 0 IFN-Y, por sua vez, estimula a atividade microbicida dos macrófagos. Os mecanismos efetores dos macrófagos incluem a produção de radicais tóxicos de oxigênio e intermediários do nitrogênio, produtos do metabolismo do ácido araquidônico pela via da 5-lipoxigenase e a degradação do triptofano intracelular do parasita. Esses eventos atuam como primeira linha de defesa na resistência à infecção pelo $T$. gondii antes do desenvolvimento da resposta imune adquirida mediada por células $T^{(12-13)}$ 


\section{IMUNIDADE ADQUIRIDA CELULAR}

A imunidade celular, mediada pelos linfócitos T, atua como mecanismo de defesa contra microrganismos que sobrevivem dentro de fagócitos ou células não-fagocíticas infectadas, onde estão protegidas de anticorpos. Em resposta à estimulação antigênica, os linfócitos T auxiliares (Th), caracterizados pela presença do marcador de superfície antígenos CD4, liberam citocinas, cuja função é estimular a proliferação e a diferenciação dos linfócitos, incluindo os linfócitos B, e dos macrófagos. Os linfócitos T citotóxicos eliminam células infectadas por vírus e outros microrganismos intracelulares que se replicam no citoplasma das mesmas ${ }^{(14)}$.

Os linfócitos Th são divididos nas subpopulações Th1 e Th2, distinguidas pelo padrão seletivo de produção de citocinas. Células de perfil Th1 secretam preferencialmente IL-2 e IFN- $\Upsilon$ e contribuem na resistência aos patógenos intracelulares ${ }^{(15)}$. O IFN- $\Upsilon$ estimula a atividade microbicida dos macrófagos, cuja forma de atuação já foi descrita acima. IL-2 induz as células citocina-ativadas de fenótipo das células NK ou células T CD8 que são citotóxicas contra células-alvo infectadas pelo T. gondii ${ }^{(11)}$. Cabe destacar que a atividade protetora das células T CD8 é mediada parcialmente através da produção de citocinas, como IFN-Y, mas um efeito citotóxico direto contra o parasita também foi relatado, assim como a citotoxicidade mediada pelo complexo de histocompatibilidade maior (MHC) tipo I contra as células infectadas pelo T. gondii(1).

As células de perfil Th2 produzem IL-4, IL-5, IL-10 e IL-13 e são responsáveis pela defesa contra infecções por helmintos e artrópodes, e por reações alérgicas. Estão associadas com "downregulation" da resposta imune celular ${ }^{(16)}$. Na infecção toxoplásmica, as citocinas de perfil Th2 podem promover a multiplicação parasitária; entretanto, elas também podem ser recrutadas para controlar a resposta imune pró-inflamatória prejudicial ${ }^{(11)}$

Um distinto grupo de células Th, as células Th17, produtoras de IL-17, foi recentemente descrito e essas células apresentam um papel crucial na indução de injúria tecidual autoimune. Embora IL-17 não seja necessária para o desenvolvimento da imunidade adquirida ao T. gondii, sua ausência impede a migração precoce dos neutrófilos para os sítios de infecção o que pode ser fatal para o hospedeiro(12).

\section{IMUNIDADE ADQUIRIDA HUMORAL}

Como parasita intracelular obrigatório, a imunidade mediada por células é a principal defesa do hospedeiro contra a infecção pelo T. gondii. De toda a forma, a infecção estimula também a produção de anticorpos lgG, IgM, IgA e IgE que, além de serem utilizados para o diagnóstico da infecção pelo parasita, parecem contribuir na primeira barreira de defesa. Taquizoítas extracelulares cobertos pelos anticorpos e complemento podem ser lisados pela via clássica do complemento ou destruídos dentro dos fagócitos. Esses mecanismos, no entanto, não oferecem proteção contra os parasitas vivos que estão no interior das células. Anticorpos como lgA secretória podem interferir na interação inicial do parasita com a célula do hospedeiro nas membranas mucosas $s^{(11,13,17)}$.

\section{Plaquetas}

As plaquetas podem apresentar um papel na defesa do hospedeiro contra o T. gondii. Na ausência de anticorpos, plaquetas humanas são citotóxicas contra os taquizoítas. Esta citotoxicidade coincide com aumento significativo na liberação de tromboxane A2 e outros metabólitos do ácido araquidônico(111).

\section{RESPOSTA IMUNE NA TOXOPLASMOSE OCULAR}

O olho é um local imunologicamente privilegiado e sua resposta imune local é suprimida para prevenir a destruição tecidual(18). Embora existam muitos estudos referentes à imunologia da toxoplasmose sistêmica, poucos estudos foram realizados com relação à resposta imune na toxoplasmose ocular humana. A patogênese do processo inflamatório na toxoplasmose ocular permanece obscura, mas diversas teorias vêm sendo levantadas na tentativa de explicar esse processo.

\section{Autoimunidade}

A contribuição da autoimunidade contra antígenos da retina no dano tecidual presente na toxoplasmose ocular é ainda controversa. Anticorpos contra antígenos da retina ${ }^{(19)}$ assim como células $T$ de pacientes com toxoplasmose ocular que proliferam in vitro frente a antígenos de retina(20-21) têm sido relatados, porém estes também são detectados em indivíduos sadios normais ${ }^{(22)}$. Estudo recente com pacientes com toxoplasmose ocular com número variado de lesões oculares sugere que a presença de uma resposta immune celular contra autoantígenos retinianos pode ser um fator de proteção contra doença ocular grave ${ }^{(23)}$.

\section{ÓxIDO NITRICO (NO) E L-TRIPTOFANO}

Estudos são controversos com relação ao papel do óxido nítrico (NO) no controle da replicação parasitária. Em modelo animal demonstrou-se aumento da inflamação na coróide, retina e vítreo em infecções crônicas sob tratamento com inibidor de NO. Entretanto, em um estudo in vitro com células do epitélio pigmentar da retina humano não se observou o papel do NO no controle da replicação parasitária. Em contraste, observou-se que o L-triptofano, aminoácido essencial para o crescimento do T. gondii, parece estar envolvido no mecanismo de controle da replicação parasitáriaa ${ }^{(19)}$.

\section{IMUNIDADE CELULAR}

Uma série de estudos foi realizada para investigar o papel das citocinas no controle da infecção ocular pelo Toxoplasma gondii, tanto em modelo animal, quanto em humanos.

\section{Citocinas em modelos animais}

O papel das citocinas na retinocoroidite toxoplásmica tem sido estudado em modelos animais, nos quais tem sido proposto que citocinas apresentam um importante papel no controle da doença. Camundongos infectados pelo T. gondii desenvolveram inflamação ocular focal e envolvimento do epitélio pigmentar da retina. O tratamento desses animais com anti-IFN- $\Upsilon$ ou anti-TNF- $\alpha$ resultou em um aumento das lesões oculares, associadas principalmente com os taquizoítas ${ }^{(24)}$. Outro estudo demonstrou, no entanto, que a estimulação de endotélio vascular retiniano de camundongos infectados com IFN- $\Upsilon$, TNF- $\alpha$ e IL-1 $\beta$ inibiu o crescimento do parasita dentro destas células ${ }^{(25)}$. Esses resultados sugerem que IFN- $\Upsilon$ e TNF- $\alpha$ são elementos cruciais no controle do crescimento parasitário, que está diretamente associado ao desenvolvimento das lesões oculares.

IFN-Y é um importante mediador inflamatório com atividades antitoxoplasma. Foram usados camundongos com deficiência da produção desta citocina como modelo de hospedeiro imunocomprometido. Uma maior carga parasitária foi observada na retina, coróide e nervo óptico dos camundongos com essa deficiência. Taquizoítas foram observados nos imunodeficientes e bradizoítas nos animais selvagens. A angiofluoresceinografia demonstrou vazamento nos capilares retinianos dos hospedeiros imunodeficientes ${ }^{(26)}$. 
A interleucina-10 (IL-10) é uma citocina com importante propriedade anti-inflamatória e imunossupressora. Para entender o papel da IL-10 na retinocoroidite toxoplásmica, foram comparados camundongos com deficiência funcional do gene desta interleucina e camundongos controles selvagens (sem qualquer deficiência) após injeção intraperitoneal do parasita. Aumento da infiltração celular e necrose foram observados no tecido ocular dos hospedeiros imunodeficientes em relação aos controles ${ }^{(27)}$.

Foi investigado também o papel da interleulcina-6 (IL-6) em olhos de camundongos infectados pelo parasita, comparando a progressão da doença e o desenvolvimento da resposta imune local em camundongos selvagens e em camundongos com deficiência da produção dessa interleucina. Os hospedeiros imunodeficientes desenvolveram uma inflamação mais grave da retina, vítreo e úvea associada com o aumento do número de parasitas ${ }^{(28)}$.

\section{Citocinas em humanos}

Realizou-se um estudo para avaliar a produção de citocinas pelas células mononucleares do sangue periférico, em resposta ao antígeno do Toxoplasma gondii em pacientes brasileiros. Os indivíduos foram divididos em quatro grupos: com toxoplasmose ocular congênita, com toxoplasmose ocular adquiri$\mathrm{da}$, soropositivos e soronegativos. Pacientes com diagnóstico de toxoplasmose ocular adquirida apresentaram maiores níveis de interleucina-1 (IL-1) do que indivíduos assintomáticos. Por outro lado, indivíduos assintomáticos secretaram significativamente mais interleucina-12 (IL-12) e IFN- $\Upsilon$ do que os indivíduos com toxoplasmose ocular adquirida. Esses dados sugerem que a resistência ao desenvolvimento de lesões oculares está associada com a habilidade de produzir IL-12 e IFN-Y, e que a susceptibilidade à ocorrência de lesões está possivelmente relacionada com uma maior produção de IL-1. Já os pacientes com toxoplasmose ocular congênita secretaram significativamente menos IL-12 e IFN- $\Upsilon$ em resposta ao antígeno parasitário do que os pacientes com a doença adquirida. A resposta diminuída ao antígeno pelas células T dos indivíduos com toxoplasmose ocular congênita sugere que as células $T$ específicas para o T. gondii podem ter sido destruídas ou "anergizadas" pela exposição ao antígeno do parasita no período pré-natal ${ }^{(29)}$.

Um estudo francês também avaliou a produção de citocinas (IFN-Y, IL-10 e IL-4) por células mononucleares do sangue periférico, após cultura, expostas ao antígeno do T. gondii. Essas células foram coletadas de indivíduos portadores de toxoplasmose ocular congênita, adquirida ou indeterminada, indivíduos com sorologia positiva, mas sem lesão ocular e indivíduos com sorologia negativa. Não foi encontrada diferença significativa na produção dessas citocinas entre os indivíduos. Sugere-se que o achado contrário ao estudo anterior ${ }^{(29)}$ se deva a diferenças em alguns fatores, como tempo de cultura das células sanguíneas, carga parasitária na cultura e carga parasitária na infecção congênita ${ }^{(30)}$.

Cultura de células do epitélio pigmentar da retina de humanos foi utilizada para investigar a resposta celular primária das células retinianas à replicação intracelular do T. gondii, através da medida dos níveis secretados de IL-1, IL-6, fator estimulador de colônias de granulócito-macrófago (GM-CSF) e molécula de adesão intercelular-1 (ICAM-1). Os níveis dessas moléculas aumentaram progressivamente com o tempo e em correlação com o número de células infectadas, assim como com a concentração intracelular do parasita, sugerindo um papel imunorregulatório dessas moléculas no processo fisiopatológico da toxoplasmose ocular ${ }^{(31)}$.
Os resultados desses estudos demonstram a importância das citocinas na modulação da resposta imune na toxoplasmose ocular e sugere que a deficiência na produção de determinadas citocinas pode acarretar um aumento das lesões oculares, associado a um maior número de parasitas.

\section{Quimiocinas}

As quimiocinas e seus receptores são importantes elementos envolvidos no recrutamento de células do sistema imune por quimiotaxia além de atuar também na ativação de leucócitos, na angiogênese, hematopoiese, doenças inflamatórias e apresentar mecanismos antimicrobianos. Elas podem ser secretadas em resposta a patógenos, dentre eles o T. gondii. Nas doenças oculares, principalmente nas uveítes, estas proteínas estão sendo identificadas como importantes mediadores da resposta inflamatória ${ }^{(32)}$.

Infecção das células de Muller com T. gondii in vitro levou a um aumento dos níveis das quimiocinas CCL2, CCL5 e CXCL8 pelas células do endotélio vascular retiniano(12).

Recentemente, um estudo do nosso grupo avaliou o nível sérico de cinco quimiocinas (CCL2, CCL11, CXCL8, CXCL9 e CXCL10) em pacientes com retinocoroidite toxoplásmica ativa e controles. A intensidade do processo inflamatório foi avaliada pela graduação da opacidade vítrea, tamanho da lesão ativa e presença de vasculite retiniana. Observou-se aumento significativo dos níveis da quimiocina CXCL8, envolvida com o recrutamento preferencial de neutrófilos para tecidos inflamados, nos pacientes. Além disso, essa quimiocina apresentou-se particularmente aumentada entre os pacientes com vasculite retiniana, com lesões de maior diâmetro e houve diminuição dos seus níveis após o tratamento. Esse estudo demonstra a importância desta quimiocina na patogênese da toxoplasmose ocular, podendo ser usado como marcador de atividade da doença ${ }^{(33)}$.

\section{Susceptibilidade genética}

Em modelos murinos, fatores genéticos do hospedeiro são críticos na determinação de susceptibilidade assim como de gravidade da toxoplasmose ocular experimental. Estes fatores genéticos podem estar dentro ou fora do complexo principal de histocompatibilidade. Em humanos a associação de genes dos antígenos de histocompatibilidade (HLA, human leukocyte antigens) e toxoplasmose ocular é insignificante ${ }^{(34)}$. Polimorfismo de genes que codificam citocinas pró-inflamatórias como TNF- $\alpha$, IL-6, IL-12 e IFN- $\Upsilon$ e citocinas anti-inflamatórias como IL-10 e TGF- $\beta$ têm sido associado a várias doenças ${ }^{(35-36)}$.

Assim, recentemente, nosso grupo investigou alguns polimorfismos de genes que codificam citocinas relacionados à resposta imune na retinocoroidite toxoplásmica: IL-10, TNF- $\alpha$ e IL-1. Ao ser analisado o polimorfismo do gene responsável pela produção da IL-10, no locus -1082, associado à menor da produção desta citocina, observou-se que a presença do alelo A está associado com a ocorrência da retinocoroidite toxoplásmica ${ }^{(37)}$. Este resultado sugere que indivíduos infectados e portadores deste polimorfismo apresentam um maior risco de desenvolverem a doença, em comparação com indivíduos infectados, mas sem o polimorfismo.

O polimorfismo do gene responsável pela produção do TNF- $\alpha$, no locus -308 G/A está associado a um aumento da produção desta citocina inflamatória. Com relação à retinocoroidite toxoplásmica, não foram encontradas associações significativas deste polimorfismo com a ocorrência da doença, ao compararmos indivíduos infectados com e sem a doença, e com a recorrência da doença ${ }^{(38)}$.

A família da interleucina 1 é dividida em IL-1 $\alpha$ e IL-1 $\beta$, que são citocinas inflamatórias e IL-RA, que é uma molécula 
anti-inflamatória. Já foi demonstrado que o polimorfismo do gene produtor da IL-1 $\alpha$, no locus -889, leva a um aumento da produção dessa citocina. Não foi observada associação entre este polimorfismo e a ocorrência da retinocoroidite toxoplásmica. Mas, em uma análise entre os pacientes com um ano ou mais anos de acompanhamento, foi observada associação deste polimorfismo com a recorrência da doença ${ }^{(39)}$. Esse resultado sugere que pacientes portadores deste polimorfismo apresentam um maior risco de recorrência da doença do que os pacientes sem o polimorfismo, devido a um aumento da produção da IL-1 $\alpha$. O polimorfismo do gene produtor de IL-1 $\beta$, no locus +3954, também está associado ao aumento da produção desta citocina inflamatória. Nesse estudo, não foram observadas associações deste polimorfismo com a ocorrência ou recorrência da doença ${ }^{(39)}$.

O polimorfismo do gene responsável pela produção do IFN-Y, no locus +874 T/A, está associado a um aumento da produção desta citocina inflamatória. Em um estudo com pacientes com retinocoroidite toxoplásmica, não foram encontradas associações significativas deste polimorfismo com a ocorrência da doença, ao compararmos indivíduos infectados com e sem a doença ${ }^{(40)}$.

\section{CONCLUSÃO}

Uma série de estudos vem confirmando a relevância de elementos da resposta imune na patogênese da retinocoroidite toxoplásmica. Parece ser necessário um controlado balanço entre as citocinas pró-inflamatórias e anti-inflamatórias na determinação da ocorrência e da gravidade da doença. Além disso, evidências recentes demonstram que predisposição genética pode ser relacionada tanto com a ocorrência, quanto com a recorrência da doença.

\section{REFERÊNCIAS}

1. Oréfice F. Uveíte: clínica e cirúrgica: texto e atlas. $2^{\underline{a}}$ ed. Rio de Janeiro: Cultura Médica; 2005.

2. Bonfioli AA, Orefice F. Toxoplasmosis. Semin Ophthalmol. 2005;20(3):129-41.

3. Bahia-Oliveira LM, Jones JL, Azevedo-Silva J, Alves CC, Orefice F, Addiss DG. Highly endemic, waterborne toxoplasmosis in north Rio de Janeiro state, Brazil. Emerg Infect Dis. 2003;9(1):55-62.

4. Montoya JG, Liesenfeld O. Toxoplasmosis. Lancet. 2004;363(9425):1965-76. Comment in: Lancet. 2004:364(9434):579.

5. Rothova A. Ocular manifestations of toxoplasmosis. Curr Opin Ophthalmol. 2003; 14(6):384-8.

6. Koo L, Young LH. Management of ocular toxoplasmosis. Int Ophthalmol Clin. 2006; 46(2):183-93

7. Rathinam SR, Namperumalsamy P. Global variation and pattern changes in epidemiology of uveitis. Indian J Ophthalmol. 2007;55(3):173-83.

8. Sibley LD, Mordue DG, Su C, Robben PM, Howe DK. Genetic approaches to studying virulence and pathogenesis in Toxoplasma gondii. Philos Trans R Soc Lond B Biol Sci. 2002;357(1417):81-8.

9. Holland GN. Ocular toxoplasmosis: a global reassessment. Part Il: disease manifestations and management. Am J Ophthalmol. 2004:137(1):1-17.

10. Vallochi AL, Nakamura MV, Schlesinger D, Martins MC, Silveira C, Belfort R Jr, et al. Ocular toxoplasmosis: more than just what meets the eye. Scand J Immunol. 2002;55(4): 324-8.

11. Roberts F, McLeod R. Pathogenesis of toxoplasmic retinochoroiditis. Parasitol Today. 1999;15(2):51-7.

12. Garweg JG, Candolfi E. Immunopathology in ocular toxoplasmosis: facts and clues. Mem Inst Oswaldo Cruz. 2009;104(2):211-20.

13. Hunter CA, Suzuki Y, Subauste CS, Remington JS. Cells and cytokines in resistance to Toxoplasma gondii. Curr Top Microbiol Immunol. 1996;219:113-25.

14. Abbas AK, Lichtman AH. Imunologia celular e molecular. $5^{\underline{a}}$ ed. Rio de Janeiro: Elsevier; 2005. p.579.
15. Mosmann TR, Coffman RL. TH1 and TH2 cells: different patterns of lymphokine secretion lead to different functional properties. Annu Rev Immunol. 1989;7:145-73.

16. Garside P, Mowat AM. Polarization of Th-cell responses: a phylogenetic consequence of nonspecific immune defence? Immunol Today. 1995;16(5):220-3.

17. Gazzinelli RT, Amichay D, Sharton-Kersten T, Grunwald E, Farber JM, Sher A. Role of macrophage-derived cytokines in the induction and regulation of cell-mediated immunity to Toxoplasma gondii. Curr Top Microbiol Immunol. 1996;219: 127-39.

18. Caspi RR. Ocular autoimmunity: the price of privilege? Immunol Rev. 2006;213: 23-35.

19. Wallace GR, Stanford MR. Immunity and Toxoplasma retinochoroiditis. Clin Exp Immunol. 2008;153(3):309-15.

20. Wyler DJ, Blackman HJ, Lunde MN. Cellular hypersensitivity to toxoplasmal and retinal antigens in patients with toxoplasmal retinochoroiditis. Am J Trop Med Hyg. 1980;29(6):1181-6.

21. Nussenblatt RB, Mittal KK, Fuhrman S, Sharma SD, Palestine AG. Lymphocyte proliferative responses of patients with ocular toxoplasmosis to parasite and retinal antigens. Am J Ophthalmol. 1989;107(6):632-41.

22. Hirose S, Tanaka T, Nussenblatt RB, Palestine AG, Wiggert B, Redmond TM, et al. Lymphocyte responses to retinal-specific antigens in uveitis patients and healthy subjects. Curr Eye Res. 1988:7(4):393-402.

23. Vallochi AL, da Silva Rios L, Nakamura MV, Silveira C, Muccioli C, Martins MC, et al. The involvement of autoimmunity against retinal antigens in determining disease severity in toxoplasmosis. J Autoimmun. 2005;24(1):25-32.

24. Gazzinelli RT, Brézin A, Li Q, Nussenblatt RB, Chan CC. Toxoplasma gondii: acquired ocular toxoplasmosis in the murine model, protective role of TNF-alpha and IFN-gamma. Exp Parasitol. 1994;78(2):217-29.

25. Brunton CL, Wallace GR, Graham E, Stanford MR. The effect of cytokines on the replication of T. gondii within rat retinal vascular endothelial cells. J Neuroimmunol. 2000;102(2):182-8.

26. Norose K, Mun HS, Aosai F, Chen M, Piao LX, Kobayashi M, et al. IFN-gammaregulated Toxoplasma gondii distribution and load in the murine eye. Invest Ophthalmol Vis Sci. 2003:44(10):4375-81.

27. Lu F, Huang S, Kasper LH. Interleukin-10 and pathogenesis of murine ocular toxoplasmosis. Infect Immun. 2003;71(12):7159-63.

28. Lyons RE, Anthony JP, Ferguson DJ, Byrne N, Alexander J, Roberts F, et al. Immunological studies of chronic ocular toxoplasmosis: up-regulation of major histocompatibility complex class I and transforming growth factor beta and a protective role for interleukin-6. Infect Immun. 2001;69(4):2589-95.

29. Yamamoto JH, Vallochi AL, Silveira C, Filho JK, Nussenblatt RB, Cunha-Neto E, Gazzinelli RT, et al. Discrimination between patients with acquired toxoplasmosis and congenital toxoplasmosis on the basis of the immune response to parasite antigens. J Infect Dis. 2000;181(6):2018-22.

30. Fatoohi F, Cozon GJ, Wallon M, Kodjikian L, Peyron F. Systemic T cell response to Toxoplasma gondii antigen in patients with ocular toxoplasmosis. Jpn J Ophthalmol. 2006;50(2):103-10.

31. Nagineni CN, Detrick B, Hooks JJ. Toxoplasma gondii infection induces gene expression and secretion of interleukin 1 (IL-1), IL-6, granulocyte-macrophage colony-stimulating factor, and intercellular adhesion molecule 1 by human retinal pigment epithelial cells. Infect Immun. 2000;68(1):407-10.

32. Gonçalves RM, Teixeira AL, Campos WR, Oréfice F. O papel das quimiocinas nas uveites. Arq Bras Oftalmol. 2007;70(2):363-70.

33. Gonçalves RM, Rodrigues DH, Camargos da Costa AM, Teixeira MM, Ribeiro Campos W, Oréfice F, et al. Increased serum levels of CXCL8 chemokine in acute toxoplasmic retinochoroiditis. Acta Ophthalmol Scand. 2007;85(8):871-6.

34. Meenken C, Rothova A, de Waal LP, van der Horst AR, Mesman BJ, Kijlstra A. HLA typing in congenital toxoplasmosis. Br J Ophthalmol. 1995;79(5):494-7.

35. Lu F, Huang S, Hu MS, Kasper LH. Experimental ocular toxoplasmosis in genetically susceptible and resistant mice. Infect Immun. 2005;73(8):5160-5.

36. Cordeiro CA, Moreira PR, Dutra WO, Campos WR, Teixeira AL, Oréfice F. [Imunogenetics in the uveitis]. Arq Bras Oftalmol. 2008;71(2):295-9. Portuguese.

37. Cordeiro CA, Moreira PR, Andrade MS, Dutra WO, Campos WR, Oréfice F, et al. Interleukin-10 gene polymorphism (-1082A/G) is associated with Toxoplasmic Retinochoroiditis. Invest Ophthalmol Vis Sci. 2008:49(5):1979-82.

38. Cordeiro CA, Moreira PR, Costa GC, Dutra WO, Campos WR, Oréfice F, et al. TNF-alpha gene polymorphism (-308G/A) and toxoplasmic retinochoroiditis. Br J Ophthalmol. 2008;92(7):986-8.

39. Cordeiro CA, Moreira PR, Costa GC, Dutra WO, Campos WR, Oréfice F, et al. Interleukin-1 gene polymorphisms and toxoplasmic retinochoroiditis. Mol Vis. 2008:14:1845-9.

40. Albuquerque MC, Aleixo AL, Benchimol El, Leandro AC, das Neves LB, Vicente RT, et al. The IFN-gamma +874T/A gene polymorphism is associated with retinochoroiditis toxoplasmosis susceptibility. Mem Inst Oswaldo Cruz. 2009;104(3):451-5. 